\title{
Cold dry air provocation is a reliable diagnostic tool in nonallergic rhinitis*
}

\author{
Yanran Huang ${ }^{1,2, *}$, , Hongfei Lou ${ }^{1,2, \#}$, Chengshuo Wang ${ }^{1,2}$, Luo Zhang ${ }^{1,2,3}$ \\ Department of Otolaryngology Head and Neck Surgery, Beijing TongRen Hospital, Capital Medical University, Beijing, China \\ Beijing Key Laboratory of Nasal Diseases, Beijing Institute of Otolaryngology, Beijing, China \\ Department of Allergy, Beijing TongRen Hospital, Capital Medical University, Beijing, China
}

Rhinology 57: 3, 225 - 230, 2019

https://doi.org/10.4193/Rhin18.187

*Received for publication:

August 19, 2018

Accepted: December 5, 2018

\#: These authors contributed equally in this paper

Background: No diagnostic criteria have been available for nonallergic rhinitis (NAR) to this point in time. Nonspecific nasal hyperresponsiveness (NHR), which could be efficiently assessed by cold dry air (CDA) provocation, is an essential feature of NAR. Thus, this study's purpose was to assess the diagnostic value of CDA provocation in discriminating patients with NAR from healthy individuals.

Methods: CDA provocation was performed among 13 healthy volunteers and 15 NAR patients. Nasal symptom scores, total nasal volume (TNV), total nasal resistance (TNR) and minimal cross-sectional area (MCA) were checked before and after the provocation. Non-paramedic tests and receiver operating characteristic $(\mathrm{ROC})$ curves were used in data evaluation.

Results: Subjects in the NAR group showed significantly larger changes after CDA provocation in total nasal symptom score (TNSS) and nasal congestion score compared with healthy volunteers, while the change for rhinorrhea score was comparable. Similarly, significant differences in the change of MCA and TNV were witnessed between both groups. We built a predictive model for NAR, $Y=-3.825+2.520^{*} \Delta T N R(\%)+1.027^{*} \Delta T N S S-6.542^{*} \Delta T N V(\%)$, whose area under curve was 0.93 . According to the Youden index, the criterion was set to be $Y>-0.52$, when its sensitivity and specificity were $93.3 \%$ and $84.6 \%$, respectively.

Conclusions: With superior patient-friendliness, safety and efficacy, we successfully performed the first NAR predictive model in the Chinese population, based on a short protocol of CDA provocation, by means of both subjective scores and objective tests (acoustic rhinometry and rhinomanometry).

Key words: acoustic rhinometry, cold dry air, nonallergic rhinitis, nasal hyperresponsiveness, rhinomanometry

\section{Introduction}

According to the latest European Academy of Allergy and Clinical Immunology (EAACI) position paper ${ }^{(1)}$, as a heterogeneous disease, nonallergic rhinitis (NAR) presents a wide range of severity and clinical symptoms. Although epidemiological data on NAR is scant, since a uniformed diagnostic criteria and international consensus of NAR have not yet been met, it is estimated to affect more than 200 million people globally ${ }^{(2)}$. It is reported that the prevalence of NAR could be $17 \%-52 \%$ in different regions ${ }^{(3-5)}$. The diagnosis of NAR is based on medical history and exclusion of positive allergen tests and rhinosinusitis.

In fact, nasal hyperreactivity (NHR) is the prominent feature of $\mathrm{NAR}^{(6)}$, lacking sensitization of specific allergen, which is a distinguished difference from allergic rhinitis (AR). A consensus from EAACI report ${ }^{(7)}$ pointed out that NHR was an abnormal reaction of nasal mucosa to stimuli meant that most people would well tolerant to. Patients with NHR would experience nasal symptoms induced by nonspecific stimuli, such as sudden temperature or humidity changes, smoke, chemical pollutants and so on. However, the diagnosis of NHR still remained unclear. Since exercise-induced asthma was first introduced by Aretaeus in the 1 st century ${ }^{(8)}$, scientists have begun to study cold dry air (CDA) and its influence on both upper and lower airways. Winter sports athletes exposed themselves to CDA for such a long 
time, nearly every day, that modification of the airway, such as hyperresponsiveness, epithelial impairment, inflammation and remodeling could be observed frequently ${ }^{(9)}$. CDA provocation was proven to be a good, safe, and tolerable test for $\mathrm{NHR}^{(10,11)}$. Recently, a review on NHR mentioned that among the five main nasal provocation tests, CDA provocation test was the most patient-friendly and investigator-friendly test, with high sensitivity and specificity ${ }^{(12)}$. Kim et al. ${ }^{(11)}$ set the criteria for NHR diagnosis to be "minimal cross-sectional area (MCA) change larger than $15.0 \%$ ", with $93.3 \%$ sensitivity and $77.4 \%$ specificity. However, no study succeeded in developing a practical diagnostic model for NAR.

Twenty years ago, Braat et al. ${ }^{(13)}$ firstly described the value of CDA provocation in discriminating NAR patients from healthy subjects, with $87 \%$ sensitivity and $71 \%$ specificity. However, the protocol of CDA provocation they used was time-consuming, lasting for 30 minutes. In 2012, Van Gerven et al. ${ }^{(14)}$ adopted a shorter procedure of CDA provocation (15 minutes) to diagnose NHR in patients with idiopathic rhinitis with assessment of peak nasal inspiratory flow (PNIF). Thus, this study was the third attempt to evaluate the relationship among CDA provocation, NHR and NAR. In view of these findings, the purpose of the present study was firstly to assess the extent of short CDA provocation in patients with NAR in the Chinese population compared with healthy individuals, with both subjective scores and objective tests (acoustic rhinometry and rhinomanometry instead of PNIF). Secondly, we aimed to develop the diagnostic model of NAR based on CDA provocation with high credibility, sensitivity and specificity.

\section{Material and Methods}

\section{Study subjects and study design}

Thirteen healthy volunteers ( 2 men and 11 women) and 15 NAR patients ( 8 men and 7 women) were enrolled into the study, from Department of Otolaryngology Head and Neck Surgery, Beijing TongRen Hospital. Healthy volunteers were adults free from any nasal symptoms, with negative allergen tests. NAR patients were diagnosed by two experienced doctors from outpatients, as defined by previous consensus ${ }^{(15)}$, according to a two-year history of rhinitis symptoms, which were related to nonspecific triggers (such as temperature, humidity and so on), negative allergen tests, normal nasal endoscopic examination (to rule out severe anatomic disorders and rhinosinusitis with/without polyps) and negative nasal cytology. Serum antigen-specific IgE was taken as an allergen test using the Phadia UniCAP System (Phadia, Uppsala, Sweden), including 10 allergens, Dermatophagoides farina, Dermatophagoides pteronyssinus, mugwort, ragweed, cat dander, dog dander, hazel, goosefoot, Blattella germanica, Penicillium notatum. In order to ensure the homogeneity of the study population, subjects with the following situations were excluded: patients with unstable diseases (including severe uncontrollable asthma), and active immunologic and systemic disease; pregnant women or women in lactation period; patients with any nasal condition that could confound the efficacy or safety assessment of the CDA provocation; and patients at risk of non-compliance. In addition, medication, including nasal or oral corticosteroids, antihistamines, nasal decongestants and so on, was stopped for more than 2 weeks before the test. Nasal symptoms, such as nasal congestion, rhinorrhea, itching and sneezing, were assessed by the self-reported visual analogue scale (VAS); ranging from 0 (asymptomatic) to 3 (very severe). Total nasal symptom score (TNSS) was also documented before and after the provocation and ranged from 0 to 12 points. Subjects' sensitivity to cold air was assessed by the subjective cold hyperresponsiveness ( $\mathrm{SCH}$ ) questionnaire ${ }^{(16)}$. Participants with grade 2 or 3 were defined to be $\mathrm{SCH}$ positive, while the others were defined as $\mathrm{SCH}$ negative.

Written informed consent was obtained from all participants prior to the study, and the protocol of our study was approved by the ethics committee of Beijing TongRen Hospital.

\section{Nasal patency}

Nasal patency was tested at a room temperature of $24 \pm 5^{\circ} \mathrm{C}$ and $70 \% \pm 10 \%$ humidity. We used Eccovision acoustic rhinometry (Hood Labs, Pembroke, USA) and the ATMO 300 Rhinomanometry (ATMOS Medizin Technik GmbH\&Co., Feldkirch, Germany) to measure the total nasal cavity volume (TNV), MCA and the total nasal resistance (TNR) at 75 Pa point. TNR was calculated according to Formula $1^{(17)}$

$\mathrm{Rt}=\mathrm{RI} \cdot \mathrm{Rr} /(\mathrm{RI}+\mathrm{Rr})(1)$

where Rt represents TNR; RI and Rr represent the nasal resistance of the left nasal cavity and right nasal cavity.

\section{CDA provocation}

All the subjects were asked to stay indoors, where room temperature was around $20^{\circ} \mathrm{C}$, for 15 minutes prior to the CDA provocation. Then, a questionnaire on basic information, and a questionnaire on $\mathrm{SCH}$, and VAS of nasal symptoms, as well as nasal patency tests (acoustic rhinometry and four-phase rhinomanometry), was completed.

CDA provocation was then performed according to the previous consensus ${ }^{(10)}$, and was basically consistent with previous studies ${ }^{(11)}$. Air that we used in the provocation was provided by an air manufacturer, met the medical-use standard and was dried and cleaned through mist separators (less than 10\% humidity). The air, which was cooled by a refrigerant air dryer to around $0^{\circ} \mathrm{C}$, was delivered to participants' noses through a continuous positive airway pressure mask with the velocity of approximate 26L/min, for about 15 minutes. Due to distinctive individual 
Table 1. Demographic and survey information of the study population.

\begin{tabular}{|c|c|c|c|}
\hline Characteristic & 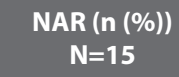 & $\begin{array}{c}\text { Control (n (\%)) } \\
\qquad N=13\end{array}$ & P-value \\
\hline Age (Mean \pm SD) & $31.33 \pm 6.97$ & $26.77 \pm 5.46$ & 0.68 \\
\hline $\begin{array}{l}\text { Gender } \\
\text { Male } \\
\text { Female }\end{array}$ & $\begin{array}{l}8(53.3 \%) \\
7(46.7 \%)\end{array}$ & $\begin{array}{l}2(15.4 \%) \\
11(84.6 \%)\end{array}$ & 0.06 \\
\hline $\begin{array}{l}\mathrm{SCH} \\
\text { Yes } \\
\text { No }\end{array}$ & $\begin{array}{l}8(53.3 \%) \\
7(46.7 \%)\end{array}$ & $\begin{array}{l}2(15.4 \%) \\
11(84.6 \%)\end{array}$ & 0.04 \\
\hline Non-smoker & 15 (100\%) & $13(100 \%)$ & 1.00 \\
\hline $\begin{array}{l}\text { Nasal burning after } \\
\text { CDA provocation } \\
\text { Yes } \\
\text { No }\end{array}$ & $\begin{array}{l}7(46.7 \%) \\
8(53.3 \%)\end{array}$ & & 0.50 \\
\hline
\end{tabular}

NAR, nonallergic rhinitis. CDA, cold dry air.

nasal resistance, the total volume of air was controlled to be 400L. Subjects were instructed to keep their mouth open, inhale through their nose and exhale through their mouth during the whole procedure.

Five minutes after CDA provocation, participants were required to fill in the questionnaires about VAS and SCH once again, and complete nasal patency tests. To ensure the homogeneity of the study, all the tests were performed by a single trained examiner.

\section{Statistical analysis}

Since data were not normally distributed, data before and after CDA provocation were compared with a Wilcoxon signed rank test. Mann-Whitney $U$ tests compared the change of TNSS and VAS, as well as the change percentage of TNV, MCA, and TNR between the NAR and control groups. Chi-square analysis was performed to analyze the relationship between $\mathrm{SCH}$ sensitivity and NAR. Logistic regression models were used to build a predictive model for incident NAR. Receiver operating characteristic (ROC) curves were used to set a diagnostic standard for NAR and evaluate its sensitivity and specificity. The area under curve (AUC) was computed and compared among the predictive model and the single indicators. In this study, we considered a value of $\mathrm{P}<0.05$ to be statistically significant. Analyses were performed using SPSS version 19.0 (IBM Corp., Armonk, NY, USA) and MedCalc version 15.2.2 (MedCalc Software, Mariakerke, Belgium).

\section{Results}

A total of 28 participants were enrolled into the study, including the NAR group ( $\mathrm{N}=15)$ and a control group $(\mathrm{N}=13)$. The basic demographic characteristics of the participants are demonstrated in Table 1. Overall, 10 (35.71\%) were male and 18 (64.29\%) female, with ages ranging from 23 to 50 years old. No significant
A

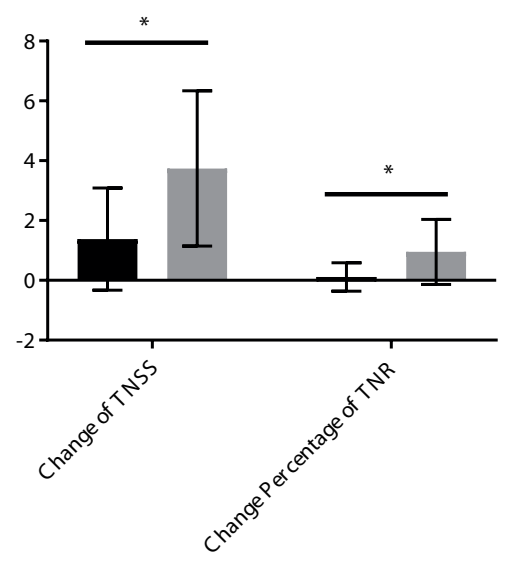

B

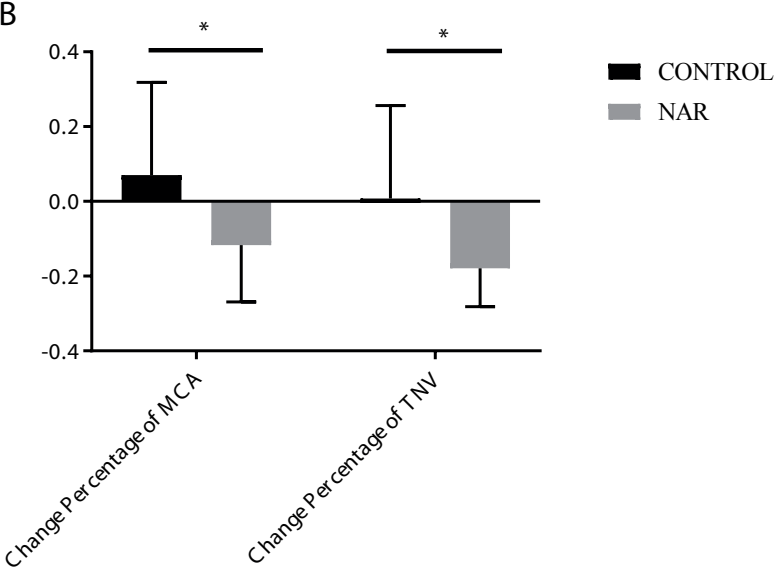

Figures 1. Change of TNSS, TNR, MCA and TNV after CDA provocation in the NAR group compared with that in the control group. Significant difference could be shown in the four factors: TNSS, total nasal symptom scores; TNR, total nasal resistance; MCA, minimal cross-sectional area; TNV, total nasal volume. Mann-Whitney $\mathrm{U}$ test, ${ }^{*} \mathrm{P}<0.05$.

difference was seen between the two groups in age $(P=0.68)$ and gender distribution ( $P=0.06)$. In the NAR group, 8 (53.3\%) patients were reported to be $\mathrm{SCH}$ positive, while 2 (15.4\%) were $\mathrm{SCH}$ positive in the control group. All the participants declared to be nonsmokers. A significant relationship was shown between $\mathrm{SCH}$ and NAR $(P=0.04)$. All the participants tolerated the procedure of CDA provocation well; no one quit the procedure because of intolerable discomfort. Besides the four nasal symptoms assessed in TNSS, the most common complaints were nasal burning $(14,50 \%)$. With good safety credits, even for the patients with significant aggravation of symptoms, all their discomforts were alleviated within 10 minutes of the procedure and vanished in half an hour.

After CDA provocation, the subjects in the NAR group showed significant changes in nasal congestion $(0.87 \pm 0.83$ vs $2.20 \pm$ $0.94, P=0.002)$, rhinorrhea ( $0.73 \pm 0.80$ vs $1.93 \pm 0.96, P=0.003)$, sneezing $(0.47 \pm 0.74$ vs $0.87 \pm 1.13, P=0.014)$, and TNSS ( 2.47 \pm 2.56 vs $5.93 \pm 2.63, \mathrm{P}=0.001$ ), compared to the baseline. No 

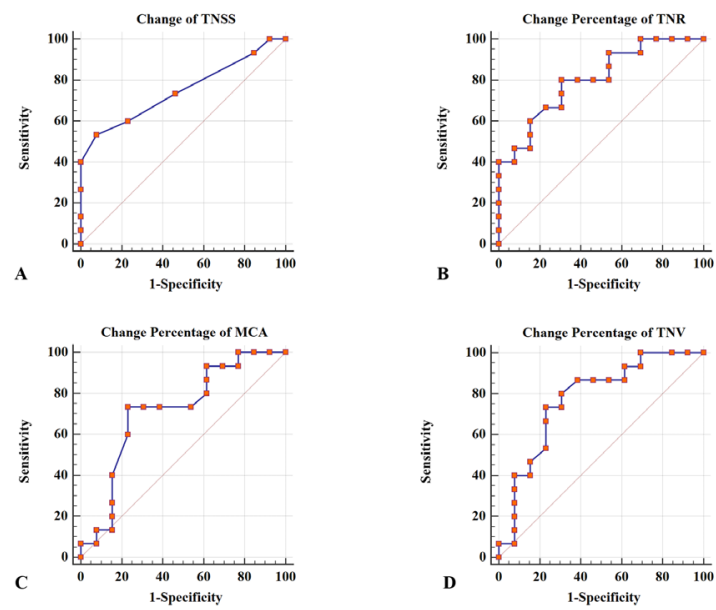

Figure 2. Receiver operating characteristic (ROC) curve of change of TNSS (A), as well as the change percentages of TNR (B), MCA (C) and TNV (D) after CDA provocation as the diagnostic criterion of NAR.

significant difference could be found in the change of nasal itching $(0.40 \pm 0.83$ vs $0.93 \pm 1.03, P=0.052)$. Further, nasal patency decreased after CDA provocation, with significant changes of TNV (16.04 \pm 3.39 vs $13.54 \pm 3.22, \mathrm{P}=0.007)$ and total nasal resistance $(0.23 \pm 0.13$ vs $0.39 \pm 0.17, P=0.009)$ compared to the baseline. However, no statistical difference could be found in the change of MCA $(0.94 \pm 0.27$ vs $0.82 \pm 0.20, P=0.202)$. In the control group, only a change in rhinorrhea was significant after the provocation $(0.38 \pm 0.51$ vs $1.54 \pm 1.05, \mathrm{P}=0.008)$.

After CDA provocation, rhinorrhea and nasal congestion were the most common symptoms in both groups, while sneezing and itching appeared less. Patients with NAR tended to have greater aggravated nasal congestion $(1.33 \pm 1.05$ vs $0.54 \pm 1.27$, $\mathrm{P}=0.022)$, sneezing $(0.40 \pm 0.51$ vs $-0.23 \pm 0.60, \mathrm{P}=0.025)$, and TNSS $(3.74 \pm 2.60$ vs $1.38 \pm 1.71, P=0.022)$, compared with the control group. Furthermore, subjects in the NAR group showed significantly larger changes in TNSS $(3.74 \pm 2.60$ vs $1.38 \pm 1.71$, $P=0.022)$ and larger change percentage of TNR $(0.95 \pm 1.10$ vs $0.11 \pm 0.47, \mathrm{P}=0.005$ ), compared with healthy volunteers ( $\mathrm{Fi}$ gure $1 \mathrm{~A})$, while the change of rhinorrhea was comparable $(1.20$ \pm 1.01 vs $1.00 \pm 1.15, P=0.618)$. Similarly, significant differences in change percentage of MCA $(-0.12 \pm 0.15$ vs $0.07 \pm 0.25, \mathrm{P}=$ $0.022)$ and TNV $(-0.18 \pm 1.10$ vs $0.01 \pm 0.25, P=0.029)$ were witnessed between both groups (Figure 1B). Significant difference in $\mathrm{SCH}$ was found between the NAR and control groups ( $\mathrm{P}=$ 0.043). Further, we compared all the results between $\mathrm{SCH}$ positive and $\mathrm{SCH}$ negative groups, finding they were all comparable. By drawing a ROC curve, we found that the change of TNSS, as well as the change percentages of TNR, MCA and TNV had value as diagnostic parameters, whose individual AUCs were 0.81 , $0.80,0.71$, and 0.77 , respectively (Figure 2 ). Since no statistical

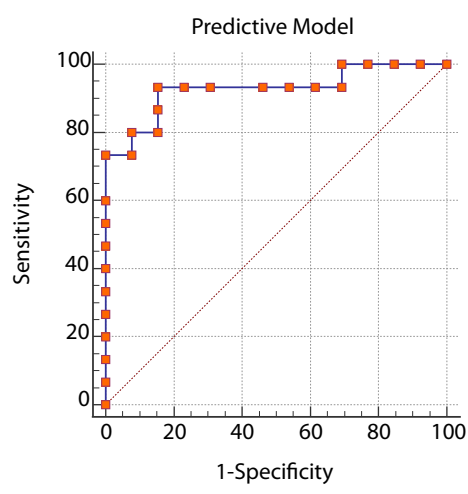

Figure 3. Receiver operating characteristic (ROC) curve of the predictive model as the diagnostic criterion of NAR.

difference could be found in the change of MCA in the NAR group, we chose the three following factors to be the optimal combination for diagnosis: change percentage of TNR and TNV, alongside with the change of TNSS. Thus, a predictive model of NAR was performed and calculated according to Formula 2:

$$
\begin{aligned}
& Y=\operatorname{Logit}(P /(1-P))=-3.825+2.520^{*} \Delta T N R(\%)+1.027^{*} \Delta T N S S- \\
& 6.542^{*} \Delta \operatorname{TNV}(\%)(2)
\end{aligned}
$$

where P represents the predictive probability of NAR; $\triangle T N R(\%)$ and $\triangle \mathrm{TNV}(\%)$ represents the change percentage ((post-provocation minus pre-provocation)/pre-provocation)) of total nasal resistance and TNV, respectively. $\triangle T N S S$ represents the change (post-provocation minus pre-provocation) of TNSS.

Further, the model was tested by ROC curve, with the AUC of 0.93 (Figure 3). According to the Youden index, the criterion was set to be $Y>-0.52$, when its sensitivity and specificity were $93.3 \%, 84.6 \%$, respectively.

\section{Discussion}

Up to now, no specific or uniformed diagnostic criteria for NAR has been made. It was reported that evaluation of nasal patency after nasal decongestion might be a helpful tool to discriminate idiopathic rhinitis from $A R^{(18)}$. In 1998, Braat et al. ${ }^{(13)}$ found that CDA provocation was superior than histamine provocation in discriminating patients with NAR from healthy individuals, with $87 \%$ sensitivity and $71 \%$ specificity. Another study shortened the duration of CDA exposure from 30 minutes to 15 minutes with satisfying results ${ }^{(14)}$. The above studies were based on a Caucasian population. Our study was the third to measure CDA provocation in NAR. We successfully provided a superior diagnostic model to previous studies, with better specificity and sensitivity in the Chinese population for the first time. Since NAR shares similar symptoms with other types of rhinitis, our model with objective examinations might be valuable in clinical practice. 
So far, the definition of CDA in such provocation studies has not been standardized, with temperature set ranged from $-39^{\circ} \mathrm{C}$, $-15^{\circ} \mathrm{C}$ to $0^{\circ} \mathrm{C}$ in 6 to 30 minutes ${ }^{(19-22)}$, or using an environmental exposure chamber ${ }^{(23)}$. In this study, we adopted the procedure explained in two international consensuses in CDA provocation $^{(7,10)}$, provoking nasal symptoms with approximately CDA of $0^{\circ} \mathrm{C},<10 \%$ relative humidity, in the velocity of $26 \mathrm{~L} / \mathrm{min}$. In our study, after CDA provocation, rhinorrhea and nasal congestion and sneezing were aggravated, together with nasal burning, while itching seemed less relevant. Nasal congestion might be associated with the turbinate swelling, and rhinorrhea with increased nasal secretion. The result was partly consistent with previous studies ${ }^{(16,21,22,24)}$. Sneezing is considered to be an irrelevant nasal symptom in most studies, which was in accordance with our findings. Kim and colleagues ${ }^{(11)}$ reported that rhinorrhea and nasal congestion were the most valuable symptoms in CDA provocation rather than sneezing and itching in the 6-minute CDA provocation. On the other hand, Van Gerven et al. reported that rhinorrhea was aggravated, and sneezing alleviated after CDA provocation in an idiopathic rhinitis group, a subgroup of $\mathrm{NAR}^{(14)}$. We could see from the published data that the idiopathic rhinitis group was relatively severer than the NAR group. What is more, since the above study was performed in a Caucasian population, the difference between races should be taken into consideration as well. The above characteristics might result in the different conclusions.

Acoustic rhinometry, rhinomanometry and peak nasal inspiratory flow (PNIF) are useful to measure nasal cavity dimensions and airflow. Van Gerven et al. adopted PNIF in their study ${ }^{(14)}$. It has been reported that PNIF is closely related to MCA of acoustic rhinometry ${ }^{(25)}$. In this study, we assessed nasal patency with acoustic rhinometry and rhinomanometry instead of PNIF. To date, most relative studies assessed the objective nasal patency with acoustic rhinometry in MCA and TNV rather than with rhinomanometry ${ }^{(11,16,22,24)}$. In this study, we included rhinometry together with the four-phased rhinomanometry into the assessment of objective nasal patency. In fact, these two above technologies are useful in evaluation of nasal function in different aspects $^{(17,26)}$. Acoustic rhinometry reflects the volume of nasal cavities at static state, while rhinomanometry reflects dynamic nasal resistance ${ }^{(27)}$. It is scientific to introduce both technologies as well as subjective symptom scores, to comprehensively assess the objective nasal patency.

Koskela ${ }^{(28)}$ held a view that CDA was more likely to be a symptom trigger, instead of the cause of developing an airway disease, considering that CDA-provoked responses could be divided into three types: the short-term response that developed within minutes; the long-term one, usually in athletes continuously exposed under CDA; and the physiological, reflexmediated lower-airway response. In this case, symptoms provoked by CDA provocation should be classified into the short-term response. However, unlike pathological responses, which could$n$ 't be treated unless medicine such as nasal decongestants or anti-cholinergic nasal sprays were used, all participants showed good tolerance, since all the symptoms significantly alleviated within ten minutes without applying any medicine. It indicated that our procedure of CDA provocation was not only efficient but safe.

As far as concerned, we compared the $\mathrm{SCH}$ sensitivity in both the NAR and control groups, finding these two were related. As a matter of fact, the SCH questionnaire was first introduced by $\mathrm{Kim}$ in $2011^{(11)}$. Then, in a series of studies that Kim et al. performed $^{(11,24)}$, they concluded that SCH grade would be precise to predict the degree of NHR, which is consistent with our results. However, no significant results could be found in all the outcomes between $\mathrm{SCH}$ positive and SCH negative groups, indicating that it was impossible to diagnose NAR by means of $\mathrm{SCH}$ alone. However, there are still limitations in the current study. Firstly, the population was small with gender disbalance. What is more, no comparison to other provocations was performed. Also, it should be noted that this study has examined only NAR and control groups, without including an AR group. As a result, these findings need to be replicated in a larger population and in a different population, and the underlying mechanism should be elucidated in the future studies.

\section{Conclusions}

In conclusion, aggravated nasal congestion and rhinorrhea could be seen after CDA provocation in the NAR population. With superior safety and efficacy, CDA provocation might be a reliable tool to help diagnose NAR from a healthy population. Some improvements to our predictive model may be brought about through additional levels in the hierarchy for more indepth studies to be carried out.

\section{Acknowledgements}

This work was supported by grants from the National Key R \& D Program of China (2018YFC0116800,2016YFC20160905200), the National natural science foundation of China (81630023, 81420108009,81400444 and 81470678), the Program for Changjiang Scholars and Innovative Research Team (IRT13082), Beijing Municipal Administration of Hospitals' Mission Plan (SML20150203), Beijing Municipal Administration of Hospitals' Youth Programme (QML20150202), and Beijing Advanced Innovation Center for Food Nutrition and Human Health (Beijing Technology and Business University (BTBU) 20181045).

\section{Authorship contribution}

$\mathrm{YH}, \mathrm{CW}$ and $\mathrm{HL}$ contributed to acquisition of data. $\mathrm{HL}$ and $\mathrm{LZ}$ provided input to study design and subject recruitment. All authors were responsible for data analysis, overall design and preparation of the manuscript. 


\section{Conflict of interest}

All authors declare no financial or commercial conflicts of inte-

rest.

\section{References}

1. Hellings PW, Klimek $L$, Cingi $C$ et al. Nonallergic rhinitis: Position paper of the European Academy of Allergy and Clinical Immunology. Allergy. 2017; 72:1657-1665.

2. Bousquet J, Fokkens W, Burney P et al. Important research questions in allergy and related diseases: nonallergic rhinitis: a GA2LEN paper. Allergy. 2010; 63:842-853.

3. Bernstein JA, Singh U. Neural Abnormalities in Nonallergic Rhinitis. Curr Allergy Asthma Rep. 2015; 15:18.

4. Chiang WC, Chen YM, Tan HK et al. Allergic rhinitis and non-allergic rhinitis in children in the tropics: prevalence and risk associations. Pediat Pulmonol. 2012; 47:1026-1033.

5. Bachert C, Van CP, Olbrecht J, Van SJ. Prevalence, classification and perception of allergic and nonallergic rhinitis in Belgium. Allergy. 2010; 61:693-698.

6. Segboer $\mathrm{CL}$, Holland $\mathrm{CT}$, Reinartz $\mathrm{SM}$ et al. Nasal hyper-reactivity is a common feature in both allergic and nonallergic rhinitis. Allergy. 2013; 68:1427-1434.

7. Bonini S, Rasi G, Brusasco V, et al. Nonspecific provocation of target organs in allergic diseases: EAACI-GA(2)LEN consensus report. Allergy. 2007; 62:683-694.

8. Randolph CC. Allergic rhinitis and asthma in the athlete. Allergy Asthma Proc. 2009; 27:104-109.

9. Sue-Chu M. Winter sports athletes: longterm effects of cold air exposure. Br J Sports Med. 2012; 46:397-401.

10. Lluch-Bernal M, MT D, E An, et al. Nasal Hyperreactivity: Nonspeci c Nasa Provocation Tests. Review by the Rhinoconjunctivitis Committee of the Spanish Society of Allergy and Clinical Immunology. J Investg Allergol Clin Immunol. 2015; 25:396-407.

11. Kim YH, Jang TY. Diagnostic criteria of nonspecific hyperreactivity using cold dry air provocation with acoustic rhinometry. Otolaryngol Head Neck Surg. 2011; 144:91 95.

12. Van GL, Steelant B, Hellings PW. Nasal hyperreactivity in rhinitis: a diagnostic and therapeutic challenge. Allergy. 2018.

13. Braat JP, Mulder PG, Fokkens WJ, van Wijk RG, Rijntjes E. Intranasal cold air is superior to histamine challenge in determining the presence and degree of nasal hyperreactivity in nonallergic non-infectious peren- nial rhinitis. Am J Respir Crit Care Med. 1998; 157:1748-1755

14. Van Gerven L, Boeckxstaens G, Jorissen M Fokkens W, Hellings PW. Short-time cold dry air exposure: a useful diagnostic tool for nasal hyperresponsiveness. Laryngoscope. 2012; 122:2615-2620

15. Kaliner MA, Baraniuk JN, Benninger MS et al. Consensus Description of Inclusion and Exclusion Criteria for Clinical Studies of Nonallergic Rhinopathy (NAR), Previously Referred to as Vasomotor Rhinitis (VMR), Nonallergic Rhinitis, and/or Idiopathic Rhinitis. World Allergy Organ J. 2009; 2:180184.

16. Kim YH, Jang TY. Nasal provocation test using allergen extract versus cold dry air provocation test: which and when? Am J Rhinol Allergy. 2013; 27:113-117.

17. Clement PA, Gordts F. Consensus report on acoustic rhinometry and rhinomanometry. Rhinology. 2005; 43:169-179.

18. Louis B, Bequignon E, Devars du Mayne $M$ et al. Nasal Compliance Measurement for Diagnosis of Idiopathic Non-Allergic Rhinitis: a Prospective Case-Controlled Study of 63 Patients. Clin Otolaryngol. 2017.

19. Le Merre C, Isber J, Chediak AD, Wanner A. Effects of cold dry air nasal stimulation on airway mucosal blood flow in humans. Arch Physiol Biochem. 2003; 111:327-329.

20. Johansson A, Bende M, Millqvist E, Bake B. Nasobronchial relationship after cold air provocation. Respir Med. 2000; 94:11191122

21. Shusterman DJ, Tilles SA. Nasal physiological reactivity of subjects with nonallergic rhinitis to cold air provocation: a pilot comparison of subgroups. Am J Rhinol Allergy. 2009; 23:475-479.

22. Kim YH, Oh YS, Kim KJ, Jang TY. Use of cold dry air provocation with acoustic rhinometry in detecting nonspecific nasal hyperreactivity. Am J Rhinol Allergy. 2010; 24:260262.

23. Bernstein JA, Salapatek AM, Lee JS, et al. Provocation of nonallergic rhinitis subjects in response to simulated weather conditions using an environmental exposure chamber model. Allergy Asthma Proc. 2012 33:333-340.

24. Kim YH, Jang TY. Nasal obstruction and rhinorrhea reflect nonspecific nasal hyperreactivity as evaluated by cold dry air prov- ocation. Acta Otolaryngol. 2012; 132:10951101

25. Kjaergaard T, Cvancarova M, Steinsvåg S Relation of Nasal Air Flow to Nasal Cavity Dimensions. Arch Otolaryngol Head Neck Surg. 2009 Jun;135(6):565-70.

26. Toh ST, Lin CH, Guilleminault C. Usage of four-phase high-resolution rhinomanometry and measurement of nasal resistance in sleep-disordered breathing. Laryngoscope. 2012; 122:2343-2349.

27. Cao HC, Zhang L. Assessment of Normal Adult Chinese Nasal Airway by Four-Phase Rhinomanometry, Rhinomanometry and Acoustic Rhinometry. Rhinology. 2010; 2010:41-44

28. Koskela HO. Cold air-provoked respiratory symptoms. International Journal of Circumpolar Health. 2007; 66:91-100

Chengshuo Wang, M.D., Ph.D.

Department of Otolaryngology

Head and Neck Surgery

Beijing TongRen Hospital

Capital Medical University

No.1, Dongjiaominxiang

DongCheng District

Beijing 100730

PR China

Tel.: (8610)58268375

Fax: (8610) 85115988

E-mail:wangcs830@126.com

Luo Zhang, M.D., Ph.D.

Beijing Institute of Otolaryngology

No. 17, HouGouHuTong

DongCheng District

Beijing, 100005

P.R. China

Tel: (8610) 65141136

Fax: (8610) 85115988

E-mail:dr.luozhang@139.com 